\title{
New antioxidant SkQ1 is an effective protector of rat neural retina under conditions of long-term organotypic cultivation
}

\author{
E. N. Grigoryan ${ }^{1,2^{\star}}$, Y. P. Novikova ${ }^{1,2}$, O. V. Kilina ${ }^{2,3}$, P. P. Philippov ${ }^{2,3}$ \\ ${ }^{1}$ Kol'tsov Institute of Developmental Biology, Russian Academy of Sciences, Moscow, Russia; \\ *Corresponding Author: e.grigoryan@hotmail.com \\ ${ }^{2}$ Belozersky Institute of Physico-Chemical Biology, Lomonosov Moscow State University, Moscow, Russia \\ ${ }^{3}$ Institute of Mitoengineering, Lomonosov Moscow State University, Moscow, Russia
}

Received 8 February 2013; revised 8 March 2013; accepted 15 March 2013

Copyright (C) 2013 E. N. Grigoryan et al. This is an open access article distributed under the Creative Commons Attribution License, which permits unrestricted use, distribution, and reproduction in any medium, provided the original work is properly cited.

\begin{abstract}
During life human eye is constantly exposed to sunlight and artificial light, the sources of reactive oxygen species (ROS)-the main cause of age-related eye pathology. A novel mitochondria-targeted antioxidant SkQ1 has recently been invented to reduce mitochondrial ROS by cleaning the mitochondria matrix, "the dirtiest place in the cell" in respect of ROS production and accumulation. Earlier we studied SkQ1 effects upon retinal pigment epithelium and choroid in the rat eye posterior cups exposed to long-term 3D organotypic culturing. It was found that under in vitro conditions 20 nM SkQ1 effectively reduced cell death in retinal pigment epithelium and choroid and protected the tissues from disintegration and cell withdrawal. In the present study we used same ex vivo conditions to examine the effect of SkQ1 upon the rat neural retina kept in the content of the posterior eye cup. Eye cups were isolated and cultured in vitro during 7,14 , and 30 days under rotation in the presence and absence of $20 \mathrm{nM}$ SkQ1 in the culture medium. Serial sections of cultivated eye cups were subjected to histology, computer morphometry and immunohistochemistry. Obtained results show that SkQ1 operates as a strong protective agent, preventing neuronal cell death and other degenerative processes in the neural retina. Cell rescue by SkQ1 was more vivid in the central part of the retina than at the periphery. That, in turn, suggests SkQ1 effectiveness in treatment of some age-related eye diseases when central part of the retina, including macula, is most susceptible to degeneration.
\end{abstract}

Keywords: Rat; Eye; Neural Retina;

Organotypic Culturing in Vitro;

Age-Related Ophthalmic Disorders;

Antioxidant SkQ1; Cell-Type Specific Proteins;

Cell Death

\section{INTRODUCTION}

Reactive oxygen species (ROS) which are effectively generated in mitochondria cause of serious damage to cells. Neural retina (NR) cells, especially rods and cones, are characterized by the intensive oxygen metabolism and high concentration of polyunsaturated fatty acids in photoreceptor membranes. These cellular features, in combination with the lifelong permanent light exposure, create conditions for extremely high risk of photoreceptors and other NR cell types' death. The tissues which underline NR, retinal pigment epithelium (RPE) and choroid inevitably respond to NR cell damage and can be injured by ROS themselves. The risk of ROS-induced retinal cell apoptosis increases with age. It is well known that just oxidative stress leads to the development of many age-related retinal diseases and visual abnormalities (AMD, glaucoma, diabetic retinopathy, etc.) [1-6].

A novel penetrating cation 10-(6'-plastoquinonyl) decyltriphenylphosphonium designated SkQ1 consists of plastoquinone conjugated with phosphonium [7]. In SkQ1, the charge of the ionized atom is distributed over a rather large molecule and, as a result, the electric forces attracting water dipoles to the ion are too weak to arrange a water layer around the ion. As a result, the cations of such type are able to penetrate into energized mitochondria and to be accumulated in their matrix [8]. This specificity of SkQ1 action is appeared very useful for the decrease of ROS generation and therefore for protection 
of tissue against cell death increasing with aging.

In a number of the in vitro and in vivo models [7,9-11] it has been demonstrated that SkQ1 possesses a powerful antioxidant activity, owing to its ability to clean the matrix space of mitochondria, "the dirtiest place in the cell" in respect of ROS [12]. Recently, we have described the protective effect of SkQ1 upon maintaining of cell morphology and viability in RPE and choroid in the content of the rat posterior eye cup under conditions of long-term, organotypic, rotary cultivation [13]. In this study, we employed a similar method to study SkQ1 effect upon NR in the content of rat posterior eye cup.

\section{MATERIALS AND METHODS}

Compound SkQ1 was synthesized as described in [7]. In our study as in the previous work [13] we used $20 \mathrm{nM}$ SkQ1, the concentration most effective in comparison with others, higher concentrations (units or hundreds of $\mathrm{nM}$ ) which were tested preliminary.

Samples of the posterior eye cups were obtained from both eyes of narcotized adult ( $2-3$ months) albino rats Wistar. All animal experiments, including maintenance, narcotization and utilization, were carried out in accordance with bioethics regulations of Russian Academy of Sciences and approved by the Laboratory Animal Care and Use Committee of the Kol'tsov Institute of Developmental Biology, Russian Academy of Science. Procedures of eye tissues isolation, rotary culturing, sample fixation as well as the methods of following preparation of cultured eye cups are described in detail in [14]. Briefly, the eye anterior segment containing the cornea, iris and lens was removed surgically. Then the remaining posterior cup of the eye consisted NR-RPE-choroid-sclera was used for rotary culturing in vitro. Obtained eye cups were put into flasks filled with $5 \mathrm{ml}$ of commercial medium DMEM containing phenol indicator, $3 \mathrm{mg}$ L-glutamine, $4 \%$ gentamicin and $10 \%$ fetal calf serum with $20 \mathrm{nM} \mathrm{SkQ1}$ or without it (6 samples in each case). Then the flasks were placed into a roller (RM-1, Elmi, Latvia) and exposed to permanent rotation $(60 \mathrm{rpm}$, $35.5^{\circ} \mathrm{C}$ ) in sterile conditions for 7, 14 and 30 days. Culturing was carried out in darkness with short time stops for checking up of eye cup samples by the artificial light. Oxygen content in flasks decreased from 20 to $5 \%$ on the 30 th day in vitro culturing. The medium was not changed during cultivation and SkQ1 was added only once, just before rotation. After the cultivation, the eye cup samples were fixed in Bouin's solution or $4 \%$ formaldehyde.

Visual and computer analyses of NR ganglion cell layer after 7-day cultivation of the eye posterior cup were performed using light microscopy (Jenaval and Ergaval, Carl Zeiss, Jena) equipped with ocular cross hairs (Carl Zeiss Jena, scale factor $0.01 \mathrm{~mm}$ ). In every forth crosssection of both, experimental (SkQ1 treated) and control samples (without SkQ1additing), a number of cells in the ganglion layer was counted inside of 400 450 squares $\left(3.125 \times 10^{-4} \mathrm{~mm}^{2}\right.$ each $)$ of the ocular cross hairs.

In addition, the sections of native, freshly isolated eye cups were analyzed in a similar way. Because of 14-day long cultivation of the complex in the absence of SkQ1 resulted in a severe destruction of the ganglion layer we didn't make a counting in it on that time point. Computer analysis was performed by the method kindly granted by A. N. Vishenskij (private communication). For this purpose, series of raster images were obtained on the $7^{\text {th }}$ day in vitro, using microscope Olimpus $\mathrm{AH} 3$ and program package Lite. The ganglion layer in the images was accentuated, using Adobe Photoshop, and the extent of the ganglion layer length was determined (in pixels) for every image. Conversion of the size of the ganglion layer in pixels to the linear size gave the value of $280-290 \mu \mathrm{m}$ for all used images. Then the tolerance was chosen, allowing us to mark precisely all nuclei in this layer. Thus, the total number of the nuclei per length unit of the layer (index of cell completeness) was evaluated. Finally, program Exel was employed for the statistical analysis of the data obtained for three series of preparations: 1 ) before culturing and 2) after it, with and without $20 \mathrm{nM}$ SkQ1.

Computer morphometric analysis of the inner nuclear and photoreceptor layers of NR after 14-day rotary culturing of the eye posterior cup. Series of 20 - 25 raster images were made for three groups of eye cup samples (before culturing and after it, with and without the addition of $20 \mathrm{nM}$ SkQ1). On NR images the inner nuclear layer was accentuated and its size was determined in pixels. Then, the equal tolerance was applied to the images of three groups of samples, allowing us to mark precisely the desolated zones of the layer, where cell lost took place. Finally, the coefficient of destruction, reflecting the ratio between "desolated zone size/whole size of the square unit of the inner nuclear layer" was determined in percentage for each image.

In photoreceptor layer, the most significant difference between the experiment (SkQ1+) and control (SkQ1-) series was detected in proximity to the outer limited membrane coupled with location area of photoreceptor inner segments. On the raster images that zone was precisely accentuated and plotted in pixels in the same way as it was done for NR inner nuclear layer. The desolated zones of the retinal portion corresponding to outer limiting membrane + photoreceptor inner segments were accentuated with the equal tolerance for three types of samples and the whole size of the zones was determined in pixels. The values obtained were used to calculate the coefficient of destruction of photoreceptor layer-the ratio between size of desolated zone and chosen square 
unit of photoreceptor layer. Finally, program Exel was employed for the statistical analysis of the data obtained for three series of eye cup samples taken before culturing and after it, with and without $20 \mathrm{nM}$ SkQ1.

Immunohistochemical analysis. Formalin-fixed samples of the eye posterior eye cup were washed with $0.1 \mathrm{M}$ phosphate buffer (PBS, pH 7.2 - 7.4, $4^{\circ} \mathrm{C}, 24$ hours), soaked with sucrose (5\% sucrose in PBS, 3 times for 20 min, and then $20 \%$ sucrose in PBS, overnight), placed in embedding medium Jung (Germany), frozen in liquid nitrogen and stored at $-20^{\circ} \mathrm{C}$. $10 \mu \mathrm{m}$ cryosections were placed on glasses (Super-frost Plus Gold, Menzel-Glazer) and stored at $-20^{\circ} \mathrm{C}$. To study the expression of NR cell type specific proteins we used following primary antibodies: rabbit polyclonal (monospecific) antibody against recoverin [15] (the dilution 1:40), mouse monoclonal antibody (1:100) against the neurofilament protein (NF-200), mouse monoclonal antibody (1:400) against glial fibrillar acidic protein (GFAP) and secondary FITCconjugated rabbit (for recoverin) and murine antibodies (all Sigma-Aldrich). Primary antibodies were diluted in $0.1 \mathrm{M}$ phosphate buffer (PBS, $\mathrm{pH} 7.4$ ), containing $0.6 \%$ triton $\mathrm{X}-100$ and $0.5 \%$ bovine serum albumin (both Sigma-Aldrich). Incubation of the sections with primary antibodies was performed overnight in a humid chamber at room temperature. Then sections were washed (3 times for $5 \mathrm{~min}$ ) with $0.1 \mathrm{M}$ phosphate buffer (PBS, pH 7.4) and incubated with secondary antibodies for 3 hours. Finally, the sections were washed with PBS, placed in a mixture (9:1) of glycerol and $0.1 \mathrm{M}$ phosphate buffer (PBS, pH 7.4). Sections treated precisely in the same way but without add of primary antibodies served as a control. Immunospecific staining was studied with microscope Olimpus AH3 equipped with green and red fluorescent channels. The latter served for control of FITC-fluorescence.

TUNEL (Terminal deoxynucleotidyl Transferase Bio-
tin-dUTP Nick End Labeling) assay was performed after 14 days of eye cup culturing using the DNA fragmentation assay kit (Promega). A number of TUNEL-labeled cells were visually evaluated on the stained sections and digital photographs made at the equal magnification (ocular $10 \times$, objective $20 \times$ ) and photography regime for SkQ1+ and control samples.

\section{RESULTS}

The analysis of NR in the content of eye posterior cups was performed after 7, 14 and 30 days of in vitro culturing in a roller, with or without SkQ1 in the medium. $20 \mathrm{nM}$ concentration of SkQ1 was used since previously it inserted positive effects on rat eye tissue viability [13].

7 days of organotypic culturing. In accordance with morphological data, NR was partially destroyed at its periphery and central region after cultivation in the absence of SkQ1. Above all, this concerned the ganglion layer which became exhausted and disintegrated. These changes were absent or much less pronounced in the presence of $20 \mathrm{nM}$ SkQ1 (Figures 1(a)-(c)). The counting of the relative number of cells per area unit $(3.125 \times$ $10^{-4} \mathrm{~mm}^{2}$ ) of the ganglion layer (Figure 2(a)) in the native (before the cultivation) NR gave the value of $2.9 \pm$ 0.7 . The value was almost 5 times less after the cultivation in the absence of SkQ1 $(0.6 \pm 0.5)$ when compared with native NR. Whereas $20 \mathrm{nM}$ SkQ1 fully prevented the ganglion layer from destruction during a week of the culturing: here, the relative number of cells, cell density in the layer was equal to $3.3 \pm 0.5$. Computer analysis (Figure 2(b)) demonstrated a similar trend: the index of the cell completeness in the ganglion layer per its extent unit $(280-290 \mu \mathrm{m})$ was estimated (in pixels) as $1733.9 \pm 444.1$ for native NR and $367.7 \pm 152.1$ or $1891.3 \pm 649.3$ for NR cultivated without or with $20 \mathrm{nM}$ SkQ1, respectively. Other layers of NR did not express

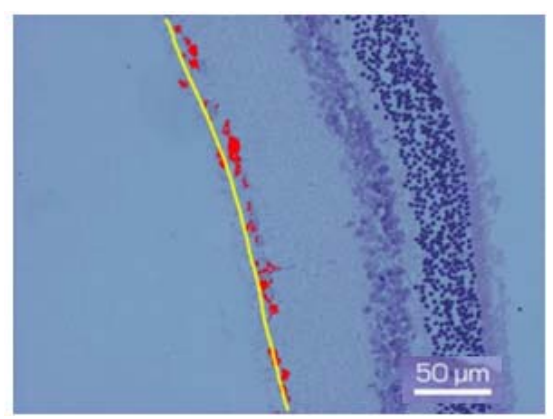

(a)

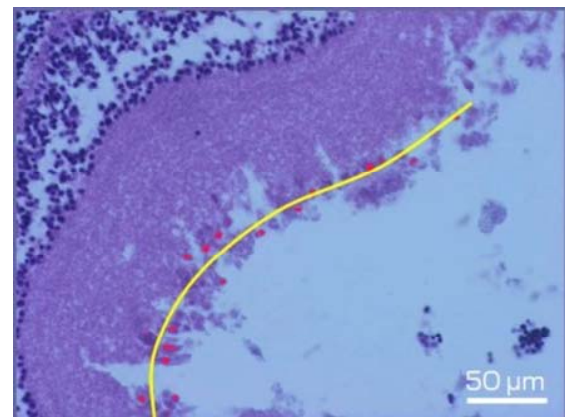

(b)

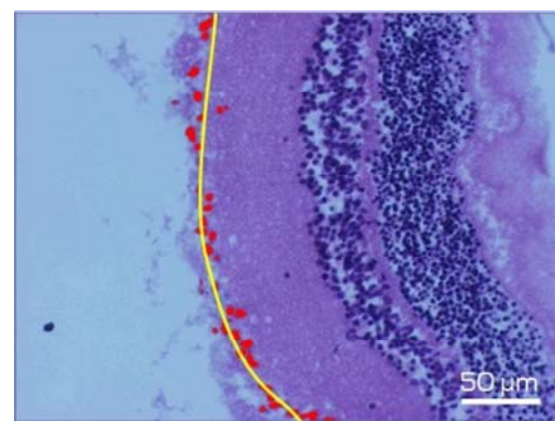

(c)

Figure 1. Fragment of the ganglion layer of NR before (a) and after 7-day organotypic cultivation of the eye posterior cup in the absence (b) or presence (c) of $20 \mathrm{nM}$ SkQ1. An example of the complex cross-sections stained with hematoxylin-eosin. In the present cases, means of the area occupied by ganglion cell bodies (red color) are 2172 (a), 665 (b), and 2393 (c) in pixels (corresponding statistical data are present in the text and in Figure 2); length of the layer (yellow lines) is $270 \mu \mathrm{m}$ (a), $280 \mu \mathrm{m}$ (b) and $294 \mu \mathrm{m}$ (c). Magnification: Ob. $20 \times$, Oc. $10 \times$. 


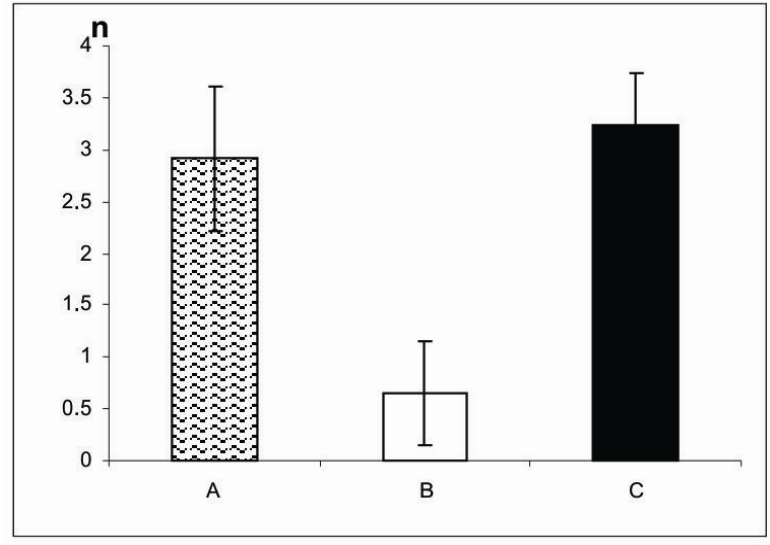

(a)

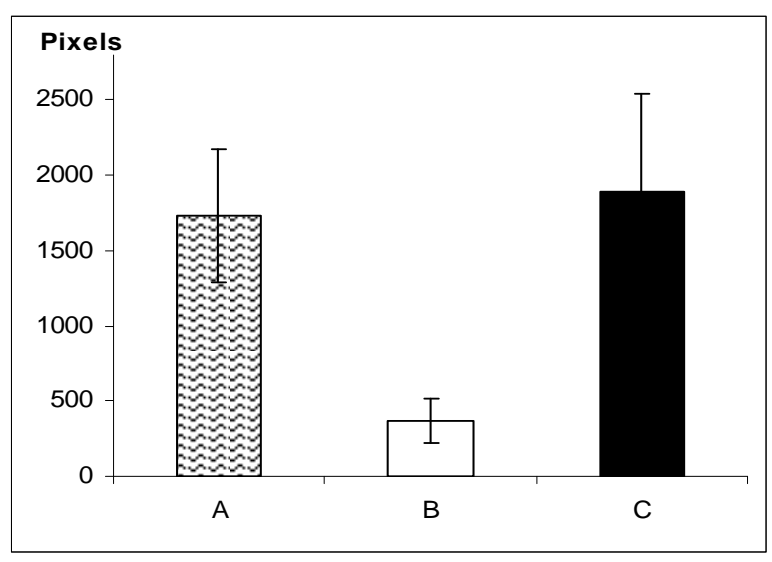

(b)

Figure 2. Relative number of cells in the NR ganglion layer before (A) and after 7-day organotypic cultivation of the eye posterior cup in the absence (B) or presence (C) of $20 \mathrm{nM}$ SkQ1, estimated by the visual (a) and digital (b) image analyses. The data presented have been obtained after statistical treatment of 30 images similar to that shown in Figure 1.

so evident changes after 7-day roller cultivation in both cases, with and without SkQ1.

An immunohistochemical study of NR performed on the $7^{\text {th }}$ day of culturing in vitro detected the expression of proteins specific for main cell types of NR (Figure 3). The neurofilament protein NF-200 in the neural processes and optic nerve, the intermediate filament protein GFAP in the end-feet of Mullerian glial cells, and the photoreceptor-specific calcium-binding protein recoverin, all were observed in rat NR after culturing in the presence of $20 \mathrm{nM} \mathrm{SkQ1.} \mathrm{At} \mathrm{the} \mathrm{same} \mathrm{time} \mathrm{NR} \mathrm{cultivated}$ without SkQ1 demonstrated only weak (NF-200 and GFAP) or no (recoverin) immunospecific staining.

14 days of organotypic culturing. Morphological pictures showed that in the absence of SkQ1 the ganglion layer, including cell bodies and long axons of ganglion cells, underwent further exhaustion and disintegration. In contrast, in the presence of $20 \mathrm{nM} \mathrm{SkQ1,} \mathrm{a} \mathrm{part} \mathrm{of} \mathrm{gan-}$ glion cell population was conserved but dead cells replaced by cells of other types: amacrine, microglial cells, and macrophages, migrated to the layer from outward (not shown). According to the computer data, the destruction coefficient calculated for inner nuclear layer of isolated NR before culturing was equal to $0.06 \pm 0.04$. Culturing for 14 days in vitro in the presence of $20 \mathrm{nM}$ SkQ1 diminished the process of cell loss in the inner nuclear layer: the coefficient of destruction in the layer averaged $0.18 \pm 0.04$ and $0.44 \pm 0.08$ with and without $20 \mathrm{nM}$ SkQ1, respectively. In other words, the protective effect of SkQ1 exceeded two times (Figures 4 and 5). TUNEL assay also revealed remarkable decrease of dying cells in the inner nuclear layer in the presence of 20 nM SkQ1 (not shown).

In the area of outer limited membrane and photoreceptor inner segments, SkQ1 effect was even more pronounced. According to the computer data, the coefficient of destruction in this area before culturing comprised $0.02 \pm 0.003$, whereas after 14 days in vitro, it was equal to $0.07 \pm 0.04$ and $0.43 \pm 0.13$ with or without $20 \mathrm{nM}$ SkQ1, respectively, i.e., a 6-fold effect of SkQ1 took place (Figures 6 and 7).

30 days of organotypic culturing. In the absence of

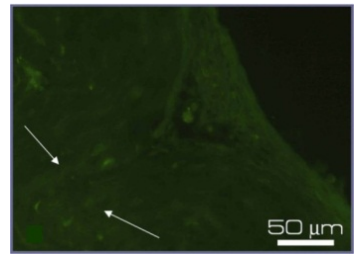

(a)

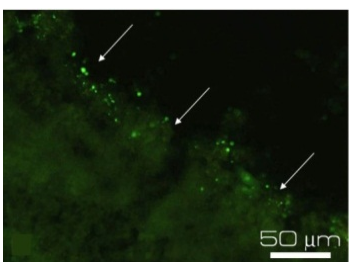

(c)

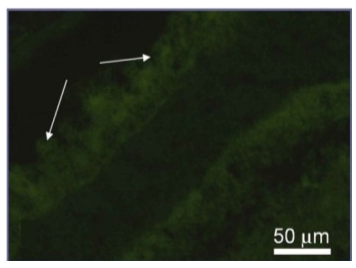

(e)

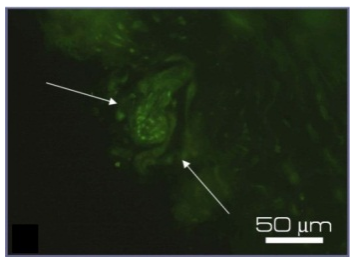

(b)

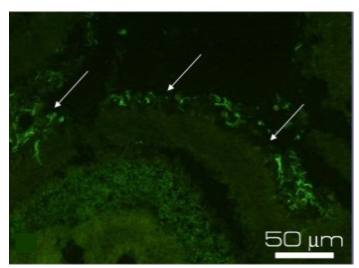

(d)

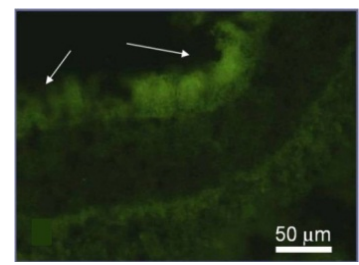

(f)
Figure 3. Immunostaining of the proteins specific for the main cell types of NR after 7-day organotypic culturing of eye posterior cup in the presence (a), (c), (e) or absence (b), (d), (f) of 20 nM SkQ1. Arrows show: NF-200 in the area of optic nerve outgrowth (a), (b), GFAP in Müller cell end-feet (c), (d) and recoverin in the layer of photoreceptor outer segments (e), (f). Magnification: Ob. $20 \times$, Oc. $10 \times$. 


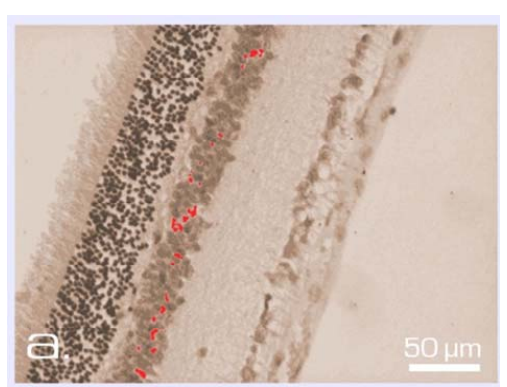

(a)

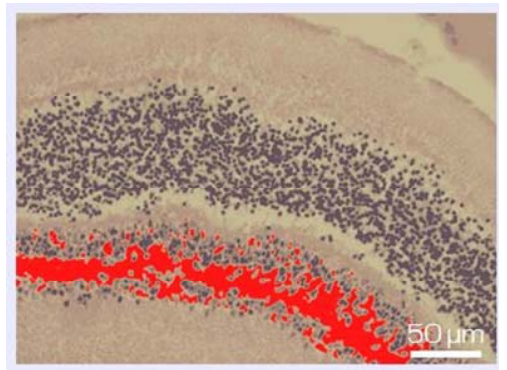

(b)

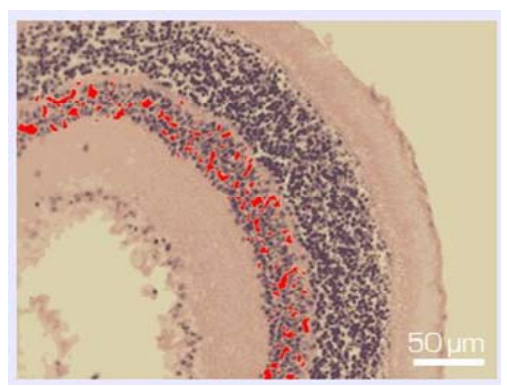

(c)

Figure 4. Inner nuclear layer of NR before (a) and after 14-day long organotypic cultivation of the eye posterior cup in the absence (b) or presence (c) of $20 \mathrm{nM}$ SkQ1. An example of the complex cross-sections stained with hematoxilin-eosin. In the present cases, relative means of the destroyed areas of the layer (red color) are 7\% (a), 45\% (b), and 17\% (c) (corresponding statistical data are present in the text and in Figure 5). Magnification: Ob. 20×, Oc. 10×.

SkQ1, almost a total destruction of NR and the death of its cells occurred. Small clusters of monocytes were present in the area of ganglion cell layer that gradually lost. In the very central zone of NR, nuclear layers along with plexiform ones were much more affected and demonstrated the death of NR cell bodies and processes. In contrast, $20 \mathrm{nM} \mathrm{SkQ1}$ protected the main stratified architecture of NR as well as the density of cells in nuclear layers and processes in plaxiform layers of this most sensitive area of NR. In this case, instead of apoptosis a translocation of NR cells was seen. Thus, the ganglion layer contained translocated amacrine cells, as well as residential macrophage and microglial cells in addition to the ganglion cells which could also survived (Figure 8).

\section{DISCUSSION}

In our previous study, we have demonstrated the protective effects of the novel penetrating cation 10-(6'plastoquinonyl) decyltriphenylphosphonium, SkQ1, upon RPE and choroid under the conditions of a long-term organotypic culturing of eye posterior cup [13]. Now we have found that $20 \mathrm{nM} \mathrm{SkQ1}$ conserves the native structure and decreases cell death of NR cultivated in the content of eye posterior cup under similar ex vivo experimental conditions. Thus, after 7 days of in vitro culturing in the absence of SkQ1 a massive death of ganglion cells was detected, while other layers of NR did not show such evident changes. After a 14-day cultivation the ganglion layer, including bodies and axons of ganglion cells, underwent a further exhaustion and disintergration; besides, significant damages and cell losses in the inner nuclear layer, outer limited membrane and photoreceptor inner segments took place by this time. Quite a different picture was seen in the presence of 20 nM SkQ1. Here, after 7 days of roller cultivation of the complex the ganglion layer remained unchanged; the same was true for the expression of the specific NR markers, such as the neuronal protein NF-200, the photoreceptor protein recoverin and glia-specific protein
GFAP. Expression of proteins, specific for main retinal cell types, suggests a good protection by SkQ1 of cellular metabolism and interactions. The ganglion layer became slightly disturbed after 14 days of the cultivation with SkQ1 due to the replacement of a small fraction of dying ganglion cells by the neighboring cells of other types. At this time $20 \mathrm{nM}$ SkQ1 also suppressed the damage to the inner nuclear layer and especially to the area of outer limited membrane and photoreceptor inner segments. TUNEL-test confirmed the ability of SkQ1 to protect cell death in these NR layers. Even after 30 days of the cultivation (extremely long time for "whole amount" culturing of any adult neural tissue), when almost the total destruction of NR and the death of its cells occurred in the absence of SkQ1, morphology and stratified structure of NR as well as the density of cell bodies in the NR nuclear layers were still partially preserved by $20 \mathrm{nM}$ SkQ1.

Therefore, one may conclude that SkQ1 maintains the

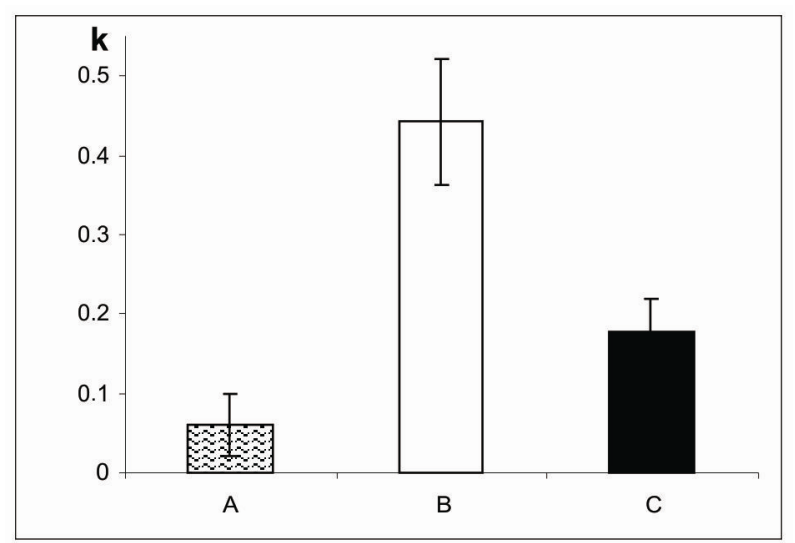

Figure 5. The destruction coefficient $(\mathrm{k})$ for the inner nuclear layer of NR before (A) and after a 14-day organotypic cultivation of the eye posterior cup without (B) or with (C) $20 \mathrm{nM}$ SkQ1, estimated by means of computer programs. The data presented have been obtained after statistical treatment of 30 images similar to that shown in Figure 4. 


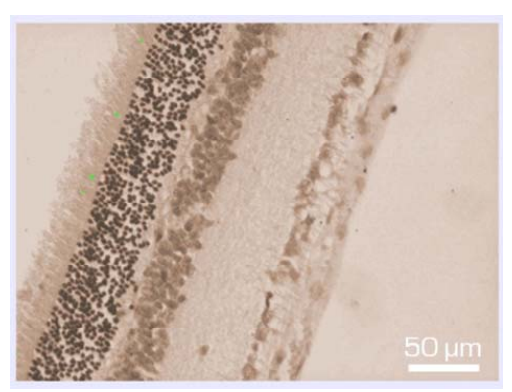

(a)

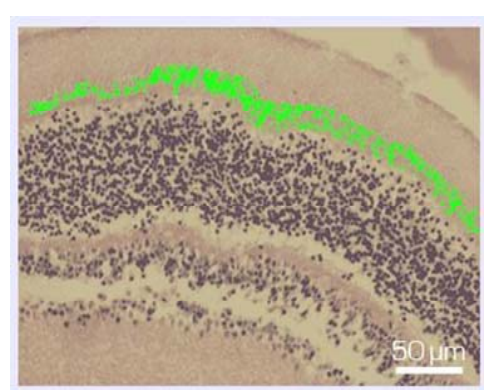

(b)

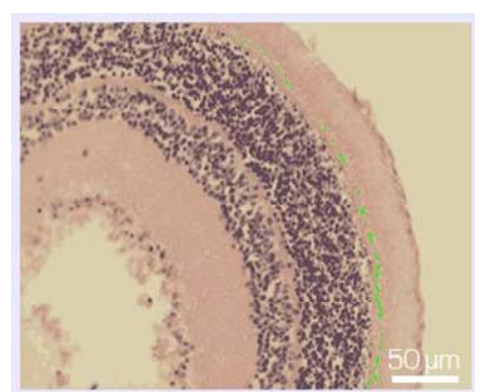

(c)

Figure 6. Zone of photoreceptor inner segments and outer limiting membrane of NR before (a) and after 14-day organotypic cultivation of the eye posterior cup in the absence (b) or presence (c) of $20 \mathrm{nM}$ SkQ1. An example of the complex cross-sections stained with hematoxilin-eosin. In the present cases, relative means of the destroyed area (green color) are 1.2\% (a), 54\% (b), and 13\% (c) (corresponding statistical data are present in the text and in Figure 7). Magnification: Ob. 20×, Oc. 10×.

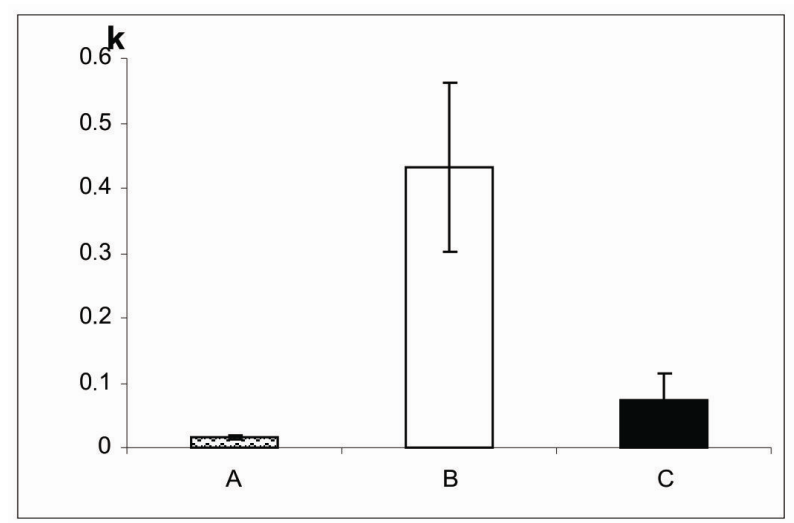

Figure 7. The destruction coefficient (k) for the region of photoreceptor inner segments and the outer limiting membrane of NR before (A) and after a 14-day organotypic culturing of the eye posterior cup in the absence (B) or presence (C) of $20 \mathrm{nM}$ SkQ1, estimated by means of computer programs. The data presented have been obtained after statistical treatment of 30 images similar to that shown in Figure 6.

viability of NR during long-term organotypic cultivation in vitro. A number of experiments made in various in vitro and in vivo models have revealed that the protective effects of SkQ1 are due to its antioxidant activity [7, 9-11]. Hence, the SkQ1 protective action upon NR in the ex vivo experimental conditions could be explained by the same cause. Irrespective of the mechanism of SkQ1 action upon NR, this compound can be considered as a potential medication for the treatment of visual disorders, underlied with oxidative damage to NR.

A dramatic example of such a disorder is age-related macular degeneration (AMD) that is the main cause of an irreversible loss of vision and blindness in people aged 60 and older. Though AMD is a complex and multifactorial disease, oxidative processes in the retina and neighboring tissues of the eye are believed to play a critical role in the AMD progression. Another example of agerelated eye pathology is glaucoma. Despite it is also multifactorial disease, aging-related oxidative stress appea- red to contribute markedly to altered regulation of immune response in glaucoma [6]. An increase of ganglion cells viability when NR cultured in the presence of SkQ1 allow us to suggest the efficacy of this antioxidant for the treatment of glaucoma as the death just of these cells is believed to be the direct reason of the disease [16].

As the optic system of the eye focuses light to the central region of the retina, macula, containing the highest density of photoreceptor cells in comparison with the periphery of the retina, even minute lesions of this region can cause a severe loss of visual acuity [1-6]. Interestingly, in our ex vivo model, the degenerative processes are also more pronounced in the center than at the periphery of NR, and the protective effect of SkQ1 is more evident in the center close to optic nerve head than in the periphery. In addition, an increase of viability of ganglion cells during long-term roller cultivation in the presence SkQ1 permits us to suggest its efficacy for the treatment of glaucoma as the death of these cells is believed to be the main cause of the disease [16].

\section{CONCLUSION}

Eye posterior cups containing the tissue complex NR-RPE-choroid-sclera were isolated from rat eyes and subjected to rotary, organotypic culturing with or without of $20 \mathrm{nM} \mathrm{SkQ1,} \mathrm{new} \mathrm{mitochondria-targeted} \mathrm{antioxidant.}$ Using histology, computer morphometry and immunohistochemistry, we have demonstrated that SkQ1 operates as a strong protective agent, preventing degenerative processes in NR and conserving the NR neurons and glia. Remarkably, degenerative processes developing in vitro in the absence of antioxidant were much more pronounced in the central part of NR than in its periphery. That resembles the in vivo situation accompanying age-related ocular diseases when the central part of the retina, macula, is particularly susceptible to degeneration. Results give us a hope for success in SkQ1 usage 


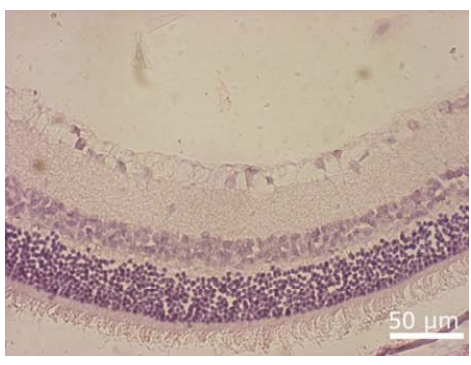

(a)

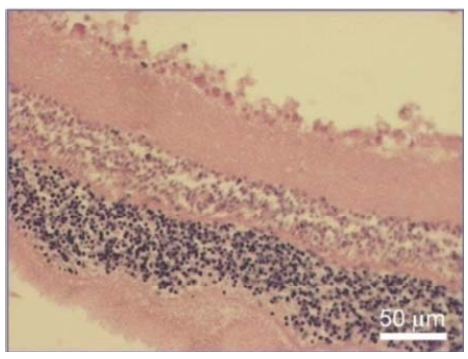

(b)

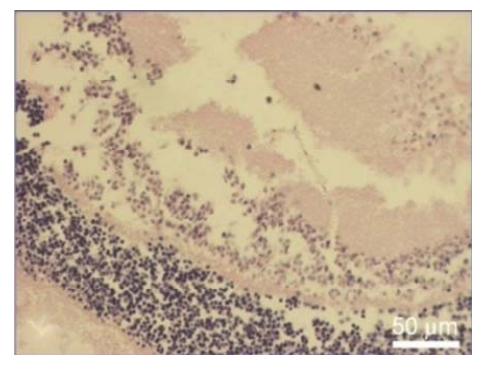

(c)

Figure 8. NR before (a) and after 30-day organotypic cultivation of the eye posterior cup in the presence (b) or absence (c) of $20 \mathrm{nM}$ SkQ1. An example of the complex cross-sections stained with hematoxilin-eosin; magnification: Ob. 20×, Oc. 10×.

for treatment of some age-related eye pathologies.

\section{ACKNOWLEDGEMENTS}

We are indebted to Prof. V.P. Skulachev for the useful discussion and to Prof. Keith Rawson-Jones and E.V. Bragina for helping us in the preparation of this article. This work was supported by the grants from the Russian Foundation for Basic Research (\#\#11-04-00125, 12-04-0 0922 and ofi-m-2011 11-04-12108)

\section{REFERENCES}

[1] Chou Newell, F.W. (1992) Ophtalmology principles and concepts. 7th Edition, Mosby-Year Book, Inc., St. Louis.

[2] Winkler, B.S., Boulton, M.E., Gottsch, J.D. and Sternberg, P. (1999) Oxidative damage and age-related macular degeneration. Molecular Vision, 5, 32-37.

[3] Tanito, M., Nishiyama, A., Tanaka, T., et al. (2002) Change of redox status and modulation by thiol replenishment in retinal photopxidative damage. Investigative Ophthalmology and Visual Science, 43, 2392-2400.

[4] Emirit, J., Edeas, M. and Brikaire, F. (2004) Neurogenerative diseases and oxidative stress. Biomed. Pharmacotherapy, 58, 39-46. doi:10.1016/j.biopha.2003.11.004

[5] Roth, F., Bindewald, A., and Holz, F.G. (2004) Keypathophysiologic pathways in age-related macular disease. Graefe's Archive for Clinical and Experimental Ophtalmology, 242, 710-716. doi:10.1007/s00417-004-0976-x

[6] Tezel, G. (2011) The immune response in glaucoma: A perspective on the roles of oxidative stress. Experimental Eye Research, 93,178-186. doi:10.1016/i.exer.2010.07.009

[7] Antonenko, Y.N., Avetisyan, A.V., Bakeeva, L.E., et al. (2008) Mitochondria-targeted plastoquinone derivatives as tools to interrupt execution of the aging program. 1. Cationic plastoquinone derivatives: Synthesis and in vitro studies. Biochemistry, 73, 1273-1287. doi:10.1134/S0006297908120018

[8] Lieberman, E.A., Topali, V.P., Tsofina, L.M., et al. (1969) Mechanism of coupling of oxidative phosphorylation and the membrane potential of mitochondria. Nature, 222,

\section{6-1078. doi: $10.1038 / 2221076 \mathrm{a} 0$}

[9] Bakeeva, L.E., Barskov, I.V., Egorov, M.V., et al. (2008) Mitochondria-targeted plastoquinone derivatives as tools to interrupt execution of the aging program. 2. Treatment of some ROS- and age-related diseases (heart arrhythmia, heart infarctions, kidney ischemia, and stroke). Biochemistry, 73, 1288-1299. doi:10.1134/S000629790812002X

[10] Agapova, L.S., Chernyak, B.V., Domnina, L.V., et al. (2008) Mitochondria-targeted plastoquinone derivatives as tools to interrupt execution of the aging program. 3. Inhibitory effect of SkQ1 on tumor development from p53-deficient cells. Biochemistry, 73, 1300-1316. doi:10.1134/S0006297908120031

[11] Neroev, V.V., Archipova, M.M., Bakeeva, L.E., et al. (2008) Mitochondria-targeted plastoquinone derivatives as tools to interrupt execution of the aging program. 4. Age-related eye disease. SkQ1 returns vision to blind animals. Biochemistry, 73, 1317-1328.

[12] Skulachev, V.P. (2005) How to clean the dirtiest place in the cell: Cationic antioxidants as intramitochondrial ROS scavengers. Life, 57, 305-310.

[13] Grigoryan, E.N., Novikova, Y.P., Gancharova, O.S., et al. (2012) New antioxidant SkQ1 is an effective protector of rat eye retinal pigment epithelium and choroids under conditions of long-term organotypic cultivation. Advances in Aging Research, 4, 31-37. doi:10.4236/aar.2012.12004

[14] Grigoryan, E.N., Novikova, Y.P., Kilina, O.V. and Philippov, P.P. (2007) New method of in vitro culturing of pigment retinal epithelium in the structure of the posterior eye sector of adult rat. Bulletin of Experimental Biology and Medicine, 4, 618-625. doi:10.1007/s10517-007-0389-z

[15] Dizhur, A.M., Nekrasova, E.R. and Filippov, P.P. (1991) New $26 \mathrm{kDa}$ protein specific for photoreceptor cells, capable of binding with immobilized delipidized rhodopsin. Biokhimiiya, 56, 225-229.

[16] Osborne, N.N. (2008) Pathogenesis of ganglion "cell death" in glaucoma and neuroprotection: Focus on ganglion cell axonal mitochondria. Progress in Brain Research, 173, 339-352. doi:10.1016/S0079-6123(08)01124-2 